\title{
LA VERITAS VITAE EN LOS ESCRITOS DE TOMÁS DE AQUINO
}

\author{
Elisabeth Reinhardt \\ Universidad de Navarra
}

\section{RESUMEN}

La expresión veritas vitae o "verdad de la vida» significa, en Tomás de Aquino, que el hombre realiza en su vida los designios existentes sobre él en la mente de Dios. El estudio de los textos aquinianos pone de manifiesto que el sintagma veritas vitae forma parte de la «triple verdad» (veritas doctrinae, vitae, iustitiae) y se sitúa a un nivel más profundo que la virtud de la veracidad. La relación entre veracidad y «verdad de la vida» abre una perspectiva interesante que puede prestarse al diálogo con los filosofos contemporáneos sobre el tema de la autenticidad.

Palabras clave: Tomás de Aquino, ética, verdad, veracidad, autenticidad.

\begin{abstract}
The expression veritas vitae or «truth of life» means, in the writings of Thomas Aquinas, the realization of each person's life according to the plan of the Creator. A study of the texts shows that the syntagma veritas vitae belongs to the so-called «three-fold truth» (veritas doctrinae, vitae, iustitiae) and is of a deeper kind than the virtue of truthfulness. For contemporary philosophers, the relationship between truthfulness and «truth of life» opens up an avenue for dialogue regarding the topic of authenticity.
\end{abstract}

Key words: Thomas Aquinas, ethics, truth, truthfulness, authenticity.

Un lector actual de los escritos aquinianos puede encontrar a su paso, sorpresivamente, respuestas válidas a inquietudes filosóficas modernas, si tiene la paciencia de penetrar en lo genuino a través de los artificios del método escolástico y si deja atrás posibles prejuicios como el de considerar el pensamiento tomasiano como un sistema cerrado en su contexto histórico, o el del «condicionante» teológico de su filosofía. No en vano se ha aconsejado aprender a «leer» a Tomás de Aquino, un consejo que contiene una promesa: la de encontrar estímulo intelectual y respuestas a interrogantes actuales y perennes del hombre $o$, al menos, sugerencias para una respuesta ${ }^{1}$.

Uno de los temas que con razón ha despertado el interés de los pensadores contemporáneos, como una laguna existente en la antropología medieval, es el de la autenticidad. Para Martin Heidegger, como se sabe, el plexo de autenticidad e inautenticidad (Eigentlichkeit-Unei-

1 En esta línea se sitúa el reciente libro de ZIMMERMANN, A., Thomas lesen, Stuttgart-Bad Cannstadt, frommann-holzboog, 2000. El mismo autor, basado en su amplia experiencia docente, confirma el interés potencial por el pensamiento de Santo Tomás en intelectuales jóvenes, cf. REINHARDT, E., Conversación en Colonia con Albert Zimmermann, en REINHARDT, E., Historiadores que hablan de la historia. Veintidós trayectorias intelectuales, Pamplona, Eunsa, 2002, p. 574-575. 
gentlichkeit) se convirtió en criterio hermenéutico fundamental de su análisis filosófico ${ }^{2}$. Otros entendieron el binomio en sentido ético, o en sentido antropológico de ser «sí mismo» que postulaba José Ortega y Gasset, mientras que Soren Kierkegaard y Rudolf Bultmann le atribuían un sentido religioso. Entre los psicólogos, Philipp Lersch se ocupó de la autenticidad como factor decisivo de la unidad de la persona ${ }^{3}$, y en el terreno de la pedagogía ha interesado, entre otros, a Hubert Henz y Víctor García $\mathrm{Hoz}^{5}$.

Desde esta perspectiva donde, según diversos enfoques, se reclamaba una reflexión existencial sobre el hombre, su lugar en el mundo y el potencial de su libertad, me llamó la atención un texto de Tomás de Aquino. En él, al hilo de su argumentación sobre la verdad, menciona - al parecer, de forma marginal- la veritas vitae del hombre, más allá de los aspectos cognoscitivos propios de la verdad. La lectura de esas escasas líneas, que citaré literalmente, me suscitó la pregunta de si el Aquinate se había planteado la autenticidad y, en caso afirmativo, cómo la entendía. Al mismo tiempo surgieron otros interrogantes: ¿Estará ya agotado el estudio de la antropología y la ética aquinianas? ¿Expondrá en algún lugar de forma sistemática la veritas en sus aspectos éticos? ¿Podrá el Aquinate aportar algo al planteamiento moderno de la autenticidad?

La última de estas preguntas es quizá la más dificultosa, ya que por su amplitud requeriría un tratamiento extenso y sistemático con el fin de obviar inexactitudes y anacronismos. Por tanto, debido al límite de estas páginas, la contestación forzosamente habrá de ser somera y en términos generales. La pregunta más asequible es, evidentemente, la penúltima, y a ella dedicaré la exposición que sigue, dejando la contestación a los demás interrogantes cuando haya obtenido los resultados del rastreo de escritos.

\section{TEXTOS Y CONTEXTO DE SANTO TOMÁS SOBRE LA «VERITAS» DEL HOMBRE}

El texto antes mencionado, que despertó mi interés por el tema, se encuentra en la primera parte de la Summa Theologiae, en la cuestión 16 que trata de la verdad. La prioridad del bien sobre la verdad parece estar justificada, arguye Tomás, porque la Ética a Nicómaco (IV, 7) incluye la veritas entre las virtudes y por tanto en el ámbito del bien. La contestación aclara el carácter analógico de veritas - según se toma en sentido general o particular-y contiene tres afirmaciones sobre la verdad en el ámbito ético: la veritas como veracidad, por la que el hombre se expresa de palabra y con los hechos tal como es; la veritas vitae, por la que el hombre realiza en su vida los designios existentes sobre él en la mente de Dios, donde se encuentra también el fundamento de la verdad de los demás seres; y la verdad de la justicia, por la que el hombre cumple su deber con los otros según el orden de las leyes ${ }^{6}$. Teniendo en cuenta la distinción entre la virtud de la veracidad y la «verdad de la vida», consideremos sucesivamente los lugares que se refieren a uno y otro aspecto.

2 Especialmente en Sein und Zeit, passim.

3 LERSCH, Ph., La estructura de la personalidad, Barcelona, Scientia, ${ }^{4} 1966, \mathrm{pp} .515-522$.

4 HENZ, H., Tratado de pedagogía sistemática, Barcelona, Herder, 1968, pp. 95; 517-521.

5 GARCÍA HOZ, V., Diccionario de Pedagogía, Madrid, Labor, 1964, pp. 818; 878.

6 S.Th. I 16, 4 ad 3: «...virtus quae dicitur veritas, no est veritas communis, sed quaedam veritas secundum quam homo in vita sua implet illud ad quod ordinatur per intellectum divinum: sicut etiam dictum est veritatem esse in ceteris rebus. Veritas autem iustitiae est secundum quod homo servat id quod debet alteri secundum ordinem legum». 


\section{a) La virtud de la veracidad}

La Ética a Nicómaco es punto de referencia frecuente para Tomás en su tratado de virtudes; concretamente el capítulo 7 del libro IV es citado en prácticamente todos los lugares que tratan de la verdad como virtud, excepto en los comentarios bíblicos, donde argumenta con textos de la Sagrada Escritura. Veamos, por tanto, en primer lugar su comentario a ese texto aristotélico. Del Estagirita asume el encuadre entre las virtudes que faciliten el trato interpersonal, en este caso la veracidad como medio entre dos extremos: la jactancia y la ironía. Consiste, pues, en manifestar la realidad propia en palabras y con los hechos, y se trata de un hábito sostenido por el amor a la verdad, por encima de criterios de utilidad, de temor o de justicia. Tomás destaca precisamente este carácter de hábito que permite mantenerse entre los dos extremos. Con Aristóteles afirma que la jactancia se opone más a la veracidad que la ironía y que ésta, usada moderadamente, da una impresión desinteresada y simpática ante los demás. Y lo mismo que el texto que comenta, critica a aquellos que se deleitan astutamente en la simulación, en cuanto pretenden mostrar que no fingen de sí algo mayor de lo que son ${ }^{7}$.

En la Secunda secundae dedica el Aquinate una cuestión completa (q. 109) a la veracidad, considerándola como una parte potencial de la justicia. Se apoya en Aristóteles para afirmar que la veracidad es una virtud, pero toma de Cicerón su relación con la justicia ${ }^{8}$. Es parte potencial, porque coincide con la justicia en algunos aspectos y difiere de ella en otros. Coincide con ella por la alteridad que supone la manifestación de lo propio a otro, y por la igualdad que establece la veracidad entre los signos y la propia realidad mostrada por ellos. En cambio difiere de la justicia respecto del débito que, en la justicia, es legal y en la veracidad tiene carácter moral, de honestidad ${ }^{9}$. Este débito moral radica, en último término, en la dimensión social del hombre, ya que los hombres se deben mutuamente la veracidad que engendra la confianza recíproca, sin la cual ninguna sociedad puede subsistir ${ }^{10}$.

La q. 109 desarrolla de modo sistemático la veracidad: su carácter de virtud, su índole específica y su relación con la justicia. La verdad por la que algo se llama «verdadero», bien por la concordancia de la realidad con el intelecto que conoce o del signo con la realidad significada, o bien entre la cosa y su regla originaria, no es virtud sino el objeto o fin de la virtud. En cambio, manifestar la verdad es un acto bueno, propio de la virtud de la veracidad (veracitas) que hace bueno al que se ejercita en ella. La specialis ratio bonitatis que hace de la veracidad una virtud específica consiste precisamente en ordenar los propios actos de tal modo que las palabras y los hechos exteriores expresen debidamente la realidad significada por ellos ${ }^{11}$. Aunque Santo Tomás, en los aspectos esenciales de la veracidad, asume fuentes no cristianas -Aristóteles y Cicerón-, la perspectiva cristiana de la moral le lleva a destacar algunos matices. La manifestación de la verdad de uno mismo es buena en general (ex genere), pero el acto virtuoso requiere también la consideración de las circunstancias para evitar que se torne vicioso, como por ejemplo la autoalabanza, aunque sea de algo verdadero, o la manifestación pú-

7 Cf. In Ethic., IV, lect. 15. Versión española: TOMÁS DE AQUINO, Comentario a la Ética a Nicómaco, traducción Ana Mallea, estudio preliminar y notas Celina A. Lértora Mendoza, Pamplona, Eunsa, 2000, pp. 173176. Del vicio de la hipocresía y de la jactancia en el contexto de la teología moral, el Aquinate había tratado ya en el comentario a IV Sent., d. 16, q. 4, a. 1 y posteriormente lo hizo con detenimiento en la II-II, qq. 111 y 112 respectivamente, dedicando a la ironía la q. 113. Como se sabe, este comentario aristotélico es contemporáneo a la IIII, de la segunda docencia parisiense. También en el contexto de la teología moral vuelve el Aquinate sobre la explicación aristotélica de la veracidad, al plantear la desviación del justo medio por exceso o por defecto, cf. II-II 109,4 .

8 Cf. II-II 109,3 s.c.

9 Cf. ibid., in c.

10 Cf. ibid., ad 1.

11 Cf. II-II 109,1 c; 2 c. Santo Tomás predica la veracidad también de Dios, incluso basándose en la definición aristotélica, en cuanto todas las criaturas son semejantes a Dios, ya que tienen su fundamento en las ideas ejemplares: cf. CG. I, c. 93; cf. In ad Rom, III, 1-5 (Marietti, n. 255). 
blica indebida de los propios pecados ${ }^{12}$. En cuanto al justo medio de esta virtud, distingue entre el objeto y el acto: el objeto de la veracidad se cumple al manifestar la verdad de uno mismo sin aumentar ni quitar nada, y esto es su justo medio; pero en cuanto al acto, el justo medio consiste en decir la verdad cuándo y cómo conviene: el exceso se produce al manifestar de modo inoportuno la propia realidad, y el defecto al ocultarla cuando corresponde darla a conocer ${ }^{13}$. Por su evidente referencia al entendimiento, el Aquinate plantea también si la veracidad es una virtud intelectual. La respuesta es clara: ciertamente la verdad en cuanto conocida pertenece al ámbito del intelecto, pero manifestarla es un acto volitivo ${ }^{14}$.

\section{b) La «veritas vitae» en el marco de la «triplex veritas»}

La expresión veritas vitae o verdad de la vida que menciona Santo Tomás en la q. 16 de la Prima pars aparece en diversos lugares de su obra, aunque no en el comentario nicomaqueo. Forma parte de la «triple verdad», a saber veritas doctrinae, veritas vitae, veritas iustitiae, que podría tener origen patrístico, ya que el propio Tomás la atribuye a San Jerónimo ${ }^{15}$. El estudio cronológico de los textos presenta variaciones respecto del contenido exacto de esta noción trimembre de la verdad. Aunque argumenta con ella, principalmente en contextos morales, parece que no pretendía exponerla de modo sistemático. Interesa, por tanto, averiguar el significado mediante la comparación de los distintos lugares.

Como la q. 109 de la Secunda secundae versa sobre la veracidad y es uno de los textos más tardíos, se presta como punto de referencia para discernir el pensamiento aquiniano al respecto. La verdad de la vida, explica Aquino, es más amplia y profunda que la virtud de la veracidad, porque es vita vera en cuanto concuerda con su regla y medida que es la ley divina, de modo similar a la «verdad de las cosas». Debido a esta concordancia, la vida humana es recta, por tanto esta verdad se llama también rectitud, y afecta a todas las virtudes como ocurre también con la sencillez (simplicitas), aunque ésta atañe más directamente a la rectitud de intención ${ }^{16}$.

La veritas iustitiae es cierta rectitud cuya regla es la ley divina, pero se distingue de la verdad de la vida en cuanto ésta se refiere a la conducta individual - la verdad por la que un hombre vive rectamente él mismo-, mientras que la verdad de la justicia consiste en guardar la rectitud de la ley en lo referente a otros, que implica también la manifestación de la verdad por motivos de justicia, como por ejemplo el testimonio en los juicios ${ }^{17}$.

La veritas doctrinae, en cambio, es la verdad que resulta de la ciencia, es decir los juicios que expresan la verdad, los resultados de las demostraciones y; en general, los enunciados verdaderos. Sin embargo está relacionada con la virtud de la veracidad en cuanto nuestra propia verdad es cognoscible y expresable, y como tal pertenece al objeto de la veracidad ${ }^{18}$.

12 Cf. II-II 109,1 ad 2.

13 Cf. ibid. ad 3; cf. In IV Sent., d. 16, q. 4, a. 1, sol. I y II, donde hace referencia a los textos aristotélicos correspondientes.

14 Cf. II-II 109,3 ad 2.

15 Cf. II-II 109, 3 s.c.; 43, 7 arg. 4 y 8 arg. 2. El origen de esta expresión no está claro. La mencionan fuentes del siglo XIII que usó Aquino: Guillermo de Auxerre (Summa aurea, III, tr. 52, cap. 5), Hugo de San Caro (In Matth. super 18,7) y la Suma Halense, Ad Claras Aquas, t. III, p. 821. Esta última remite a San Jerónimo, aunque en sus escritos no se encuentra; quizá se remonte a los Decretales de Gregorio IX (cf. Deutsche Thomas-Ausgabe, Bd. 17B, Walberberg, 1966, p. 375, y ed. Marietti, II-II 43, 7 arg. 4, nota 4).

16 II-II 109, 2 ad 3: «...veritas vitae est veritas secundum quam aliquid est verum, non veritas secundum quam aliquis dicit verum. Dicitur autem vita vera, sicut etiam quaelibet res, ex hoc quod attingit suam regulam et mensuram, scilicet divinam Iegem, per cuius conformitatem rectitudinem habet. Et talis veritas, sive rectitudo, communis est ad quamlibet virtutem». Cf. I 17, I c: la voluntad libre puede apartarse del orden normativo que existe en la mente divina, por lo que la Sagrada Escritura llama los pecados «falsedades» y «mentiras»; en cambio, el obrar virtuoso se denomina veritas vitae, en cuanto se somete a la mente divina.

17 Cf. II-II 109,3 ad 3.

18 Cf. ibid. 
En el comentario al cuarto libro de las Sentencias se encuentran varios textos sobre la triple verdad, así como referencias al capítulo 7 del cuarto libro de la Ética a Nicómaco, aunque el comentario tomasiano a este texto aristotélico es mucho más tardío (cf. nota 7). Al tratar el problema del escándalo, Santo Tomás plantea si es lícito apartarse de la verdad o rectitud moral para evitar que otros se escandalicen. En la contestación parte del principio de que apartarse de la verdad es pecado y explica que esta «verdad» consiste en que el hombre adapta su conducta a la regla de la ley divina en la dimensión del conocer y del obrar. La concordancia del conocimiento con la ley divina es la veritas doctrinae; la del obrar, considerada individualmente, es la veritas vitae, y en lo referente a los deberes con otros es la veritas iustitiae ${ }^{19}$. Es el único texto en el que la rectitud general de la vida implica la triple verdad y ésta viene a ser uno de los aspectos de esa rectitud. Generalmente la triple verdad es considerada como criterio para discernir el obrar bueno, como manifiesta la expresión salva triplici veritate ${ }^{20}$; otras veces, el criterio es la veritas vitae o la veritas iustitiae ${ }^{21}$.

En otro lugar del comentario a las Sentencias, la triple verdad se expone sin referencia directa al orden establecido por Dios, sino siguiendo el texto aristotélico ya mencionado y, en lo referente a la justicia, el criterio de Cicerón. El contexto es, dentro de la escatología, la justicia y la misericordia de Dios. Partiendo de la noción de verdad como adecuación entre intelecto y cosa, afirma Santo Tomás que en esto consiste la ratio veritatis, que se puede aplicar también a las acciones humanas. Estas se relacionan con la disposición interior de la mente como el signo con lo significado y como lo regulado con la regla. Así, la concordancia del signo con lo significado es la veracidad de la que habla la Ética a Nicómaco. En cambio, la adecuación de los actos externos a las razones de justicia que están en la mente, de modo que se adapten a la regla de igualdad objetiva propia de la justicia, es la veritas iustitiae. Pero esta adecuación reguladora es aplicable no sólo a este aspecto, sino a todos nuestros actos, de modo que concuerden con la recta razón, y ésta es la veritas vitae que consiste en actuar cada uno conforme al dictamen de la recta razón. La veritas doctrinae, a su vez, es la verdad del enunciado que sigue a la adecuación entre intelecto y $\cos \mathrm{a}^{22}$. En lo que concierne la veritas vitae, aquí no se menciona la ley divina como regla de nuestros actos, pero está presente de modo implícito, tal como entiende Santo Tomás la recta razón ${ }^{23}$.

En el comentario a los salmos, cronológicamente el último de los textos tomasianos sobre la «triple verdad», las referencias son todas bíblicas. El contexto es Ps 11, 1: quoniam diminutae sunt veritates a filiis hominum. Uno de los modos de hablar de «verdades» en plural es la «triple verdad creada que está en los santos». Estas verdades no disminuyen por sí mismas,

19 In IV Sent., d. 38, q. 2, a. 4, sol. I: «Quicumque autem veritatem relinquit, peccat. Veritas autem de qua loquimur, consistit in hoc quod homo in dictis et factis suis rectitudini divinae, sive divinae legis regulae, se conformet: cui quidem homo conformari debet in his quae ad cognitionem pertinent, et hoc pertinet ad veritatem doctrinae; et in his quae ad actionem spectant; sive ea debeat aliquis per se ipsum agere, quod pertinet ad veritatem vitae, sive ea debeat $a b$ aliis observanda promulgare, quod pertinet ad veritatem iustitiae, quae consistit in rectitudine iudicii».

20 Cf. ibid., sol. II; II-II 43, 7, arg. 4, y 8, arg. 2.

21 Cf. In IV Sent., d. 38, q. 2 , a. 4, sol. II; II-II 43,8 ad 2.

22 Cf. In IV Sent., d. 46, q. 1, a. 1, sol. III. Posteriormente, la Summa Theologiae I 21, 2, al comparar justicia y verdad en Dios, parece identificar veritas iustitiae con lo que en otros lugares se llama veritas vitae, sin embargo no existe contradicción, porque parte de la base de que la ley eterna como regla de nuestras acciones se funda en la Justicia de Dios, que es la Verdad como fundamento de toda verdad, y llama veritas iustitiae la concordancia de nuestras acciones con esta ley. En el mismo artículo distingue esta veritas iustitiae (o veritas vitae, según otros textos) de la veracidad aristotélica: ésta última es la concordancia entre signo y lo significado, pero no la conformidad del efecto respecto de su causa y regla como sucede en la «verdad de la justicia» (ad 2). También en Super Ioann. 8, lect. VI (Marietti, n. 1245) emplea veritas iustitiae en el sentido de verdad de la vida: «Veritas iustitiae, quando scilicet quis agit quod convenit sibi secundum ordinem suae naturae».

23 I-II 71, 6 c: «Regula autem voluntatis humanae est duplex: una propinqua et homogenea, scilicet ipsa humana ratio; alia vero est prima regula, scilicet lex aeterna, quae est quasi ratio Dei». 
sino que se corrompen por las culpas de los hombres ${ }^{24}$. Aunque se trata de una reportación, el texto enriquece la noción de la triple verdad, y concretamente la veritas vitae, ya que destaca la rectitud de conciencia que requiere.

La verdad de la vida consiste, positivamente, en el obrar recto, es decir según la recta razón que, en último término, se fundamenta en la ley divina; y negativamente, en evitar el pecado. ¿Qué papel juega en este caso lo que no es mandato sino consejo? Esta cuestión aparece en algunos textos tomasianios en relación con la veritas vitae, o con la triple verdad. Así, por ejemplo, las obras de misericordia pertenecen a la veritas vitae ${ }^{25}$. En otro lugar afirma Santo Tomás que la triple verdad no sólo comprende lo que es necesario para la salvación, sino también aquello que permite alcanzarla de modo más perfecto ${ }^{26}$.

\section{LA VERACIDAD Y LA «VERITAS VITAE» EN RELACIÓN CON LA DEFINICIÓN DE LA VERDAD}

Los textos que se acaban de exponer contienen a veces referencias a la definición de la verdad. La más conocida es, sin duda, la que entiende la verdad como adecuación entre intelecto y cosa, por lo que el Aquinate la cita literalmente y hace diversas aplicaciones analógicas. Aunque se suele atribuir esta definición a Santo Tomás, en realidad no es suya y, además, maneja varias definiciones clásicas que compara entre sí según los aspectos que resalta una u otra ${ }^{27}$. Esta amplitud de criterio se percibe, por ejemplo, en el primer artículo de la q. 16 de la Prima pars, que versa sobre la «verdad ontológica». El Aquinate distingue entre la relación de la cosa con el intelecto del que depende en el ser (per se) - las cosas naturales dependen del entendimiento divino- y la relación de la cosa con el intelecto por el que puede ser conocido (per accidens), para concluir que la verdad está principalmente en el intelecto en cuanto su término, y secundariamente en las cosas en cuanto su origen. Hecha esta distinción, agrupa las definiciones clásicas conforme a los factores del conocimiento de la verdad, a saber, el intelecto, las cosas, y ambos a la vez. Así, la primera de las dos definiciones agustinianas citadas en ese lugar (veritas est qua ostenditur id quod est) y la de San Hilario (verum est declarativum aut manifestativum esse) corresponden a la verdad en cuanto está en el intelecto. En cambio, a la verdad de las cosas aplica Santo Tomás tres definiciones: la otra definición agustiniana (veritas est summa similitudo principii, quae sine ulla dissimilitudine est), la de San Anselmo (veritas est rectitudo sola mente perceptibilis) y la de Avicena (veritas uniuscuiusque rei est proprietas sui esse quod stabilitum est ei). $\mathrm{Y}$ a ambos, intelecto y cosa, refiere la definición que él atribuye a Isaac Israeli (veritas est adaequatio rei et intellectus) ${ }^{28}$.

24 Cf. In Psalm XI, 1-3:«Vel dicendum quod dicit veritates, propter triplicem veritatem creatam quae est in sanctis: scilicet vitae, de qua Isa. 38, 3: Memento quomodo ambulaverim coram te in veritate. Doctrinae. Matth. 12, 16: Scimus quia verax es, et viam Dei in veritate doces. Et iustitiae: de qua Exod. 18, 21: Provide de omni plebe viros potentes et timentes Deum, in quibus sit veritas. (...) Et quidem veritas vitae diminuitur, quando bonum iudicatur malum. Doctrinae, quando lux dicitur tenebrae. Veritas vero iustitiae, quando amarum iudicatur dulce». Estas expresiones están tomadas de Is 38, 20 que Aquino cita a continuación.

25 Cf. In IV Sent., d. 39 , q. 2, a. 4, qla. 2.

26 Cf. II-II 43, 7 ad 4; In IV Sent., d. 38, q. 2, a. 4, sol. II. El Aquinate especifica que lo que es materia de mayor perfección puede omitirse, salvo cuando exista una circunstancia que lo haga obligatorio, como por ejemplo el voto o el cargo.

27 Este tema ha sido estudiado por PONFERRADA, G. E., «El tema de la verdad en Santo Tomás: sus fuentes», en Sapientia, 41 (1986), pp. 11-36. El autor muestra la evolución de los escritos tomasianos en este punto y afirma que la exposición de las fuentes está más nítida y exacta en la Summa Theologiae.

28 Cf. I 16, 1 c; Verit. I, 1 c. La famosa definición parece remontarse a la Metafísica de Avicena con una remodelación posterior por teólogos parisinos del siglo XIII, según recientes estudios que recoge R. A. Gauthier en la edición leonina del comentario a la Ética, p. 252, nota a lín. 91-92. 
Si relacionamos estas definiciones con los textos expuestos anteriormente, es evidente que Aquino acude con frecuencia a la última de ellas, que aplica tanto a la veracidad como a la «triple verdad» y particularmente a la verdad de la vida. Se puede afirmar que implícitamente tiene presente también las otras definiciones. Así, las que asigna a la verdad en el intelecto se relacionan con la veritas doctrinae, y entre las definiciones que se refieren a la verdad de las cosas, la anselmiana es particularmente aplicable a la veritas vitae, porque el Aquinate entiende la rectitudo como concordancia con el origen (nam rectum est quod principio concordat).

Además de relacionar la veritas vitae con esas definiciones, es obvio que la «verdad» del hombre en su conducta es una manifestación de la verdad práctica, en su aspecto de agere. La verdad práctica en este sentido es, según Aquino, la concordancia entre el apetito recto y la razón en relación con el fin ${ }^{29}$. En su función de indicar precisamente el justo medio, la verdad práctica guía el obrar y discierne los medios adecuados para el fin. El intelecto en su función práctica y la voluntad en su capacidad de decisión son perfeccionados para ello por la virtud de la prudencia ${ }^{30}$. Por tanto, para lograr una noción completa de la veracidad y la verdad de la vida, sería preciso estudiar el tema también desde la perspectiva de la verdad práctica y de la prudencia, un cometido que excedería el marco de esta reflexión ${ }^{31}$.

\section{SÍNTESIS CONCLUSIVA}

En síntesis, se puede decir que la veracidad como virtud es la concordancia habitual, en nuestra conducta, entre los signos - palabras y hechos-y lo que somos (relación signum-signatum). Es una virtud que tiene un sentido social, porque se basa en la alteridad y, al facilitar el trato interpersonal y la confianza mutua, resulta imprescindible para la sociedad misma. En cambio, la veritas vitae es la concordancia entre los actos de cada persona con una regla o ley (relación regulatum-regula), en un doble nivel: de modo próximo y homogéneo, con la recta razón; de modo absoluto y fundante, con la ley que existe en la mente divina en cuanto causa eficiente, ejemplar y final de todo lo creado. Es una concordancia condicionada en cierto modo por la libertad del hombre que le posibilita aspirar a una perfección cada vez mayor, o renunciar a ella. Según Aquino, la veritas vitae se puede incluir o no en la clásica «triple verdad», pero en cualquier caso guarda relación con la veritas doctrinae y la veritas iustitiae. A su vez, tanto la veracidad como la verdad de la vida se relacionan con las distintas definiciones clásicas de la verdad que utiliza Santo Tomás, particularmente la de la adecuación, y presupone la verdad práctica.

Podemos retomar ahora los interrogantes formulados al principio, aunque en distinto orden. La pregunta sobre una exposición sistemática de la veritas ética, recibe una respuesta afirmativa respecto de la veracidad. En cambio, la veritas vitae está presente -de forma implícita, al menos- - en los planteamientos de la teología moral. No se sabe si el Aquinate tenía previsto un tratamiento sistemático de este aspecto de la verdad. En cualquier caso, el doble tema de la veracidad y de la verdad de la vida abre una perspectiva interesante de la antropo-. logía y ética tomasianas a la que hasta el momento se ha prestado escasa atención. Por ello es lícito pensar que el estudio de la antropología y la ética aquinianas no está aún agotado.

29 La exposición de este tema se encuentra en el Libro VI de la Ética a Nicómaco (Bk 1139a15-b13), y en el comentario tomasiano.

30 Este tema ha sido estudiado por GARCÍA-HUIDOBRO, J., «Sobre la verdad práctica en Tomás de Aquino», en Sapientia, 50 (1995), pp. 243-262. La relación entre verdad teórica y verdad práctica, sindéresis y prudencia está expuesta magistralmente por PIEPER, J., El descubrimiento de la realidad, Madrid, Rialp, 1974, pp. 90-98.

31 García López considera en esta perspectiva la veracidad y la verdad de la vida - que algunos, dice, prefieren liamar «autenticidad»-, citando el texto de Santo Tomás In IV Sent., d. 46, q. 1, a. 1, sol. III, cf. GARCÍA LÓPEZ, J., «Verdad», I, GER, 23, Madrid, Rialp, ${ }^{6} 1991$, pp. 424-426. 
Abordemos, por último, la respuesta a la pregunta más compleja: ¿La enseñanza tomasiana sobre la «verdad del hombre» se prestaría a un diálogo con el pensamiento contemporáneo acerca de la autenticidad? Es evidente que el Aquinate se había planteado la autenticidad en diversas perspectivas, aunque no utiliza este término sino el de veritas, en sentido analógico. $\mathrm{Si}$ por autenticidad entendemos la manifestación genuina de uno mismo, sin falsear la propia realidad, coincide con la veracidad. Aunque la autenticidad puede entenderse en un sentido puramente psicológico, es evidente que tanto Aristóteles como Tomás le confieren un sentido ético al considerarla como virtud. En cambio, si autenticidad es fidelidad al original, coincide con la verdad de la vida, que no se encuentra en la Ética a Nicómaco sino que proviene de la visión cristiana del hombre y tiene un fundamento ontológico inscrito en la doctrina de la creación. La autenticidad entendida de este modo sería precisamente inautenticidad para los filósofos contemporáneos de inspiración heideggeriana, ya que la recta razón y su fundamento divino, al ser un elemento heterónomo, constituiría una amenaza para la autonomía del hombre y su libertad que, entendida como pura espontaneidad, quedaría falseada por la referencia a la «recta razón».

Como señala Forment, la ética tomasiana es ciertamente teocéntrica, pero no heterónoma sino en cierto modo «personalista», porque los preceptos de la ley natural, que son evidentes por sí mismos, sintonizan con la inclinación natural del hombre y se aprehenden naturalmente como buenos $^{32}$. «La moral de Santo Tomás, fundamentada en su metafísica, es una equilibrada síntesis, anterior a las modernas antítesis entre autonomía y heteronomía, o entre moral formal y moral material. No es por ello ni una concepción anticipada, ni una superación 'dialéctica' de las escisiones y enfrentamientos modernos. Su concepción ética es totalmente coherente con su teocentrismo y personalismo, negados en la modernidad y en la postmodernidad $\diamond^{33}$. Como he advertido al principio, la discusión de este tema se antoja interesante, pero aquí sólo puede ser planteada. Tal vez, debido a esa «equilibrada síntesis», las raíces profundas y la apertura a la historia características del pensamiento tomasiano sobre el hombre, puede constituir una base de diálogo con los pensadores modernos y postmodernos. Se puede adelantar, a la vista de los textos, que la «autenticidad» en los distintos niveles señalados por Santo Tomás es personalista, porque defiende la autonomía, núcleo de la libertad que para él consiste fundamentalmente en que el hombre es «dueño de sus actos» ${ }^{34}$, con la causalidad propia que le corresponde por ser imagen de Dios. A pesar de su dependencia ontológica de Dios - mejor, gracias a ella-, el hombre es verdaderamente «autónomo» en su obrar, porque la libertad le hace, en cierto modo, estar «solo» con sus actos, los valores y criterios que los motivan y la posible repercusión sobre los otros. Un vivir profundamente verdadero, comparable con la auténtica «soledad» que propone Joaquín Lomba como un desideratum, en contraste con la soledad angustiosa de quien se cierra sobre sí mismo: «la soledad que surge de la libertad y del tiempo internos de la conciencia hace que los hombres, en primer lugar, hallen su identidad, encontrándose, en consecuencia, a gusto consigo mismos, y, en segundo lugar, esa auténtica y gloriosa soledad hace que los seres humanos se puedan amar desde lo más profundo de su propia mismidad» ${ }^{35}$.

\section{Elisabeth Reinhardt \\ Instituto de Historia de Iglesia. Universidad de Navarra \\ 31080 Pamplona \\ E-mail: erein@unav.es}

32 Cf. FORMENT, E., «El personalismo de Santo Tomás», en Sapientia, 45 (1990), p. 294.

33 Ibid.

34 Cf. I-II, pról.

35 LOMBA, J., «El urgente reto de lo misterioso. Reflexiones en torno al espacio, al tiempo y a la libertad», en Themata, 9-10 (1992), p. 236. 